\title{
Complement inhibition initiated recovery of a severe myasthenic crisis with COVID-19
}

\author{
Ulrich Hofstadt-van Oy ${ }^{1,4}\left(\right.$ D $\cdot$ Slobodan Stankovic ${ }^{1} \cdot$ Corinna Kelbel $^{2} \cdot$ Daniel Oswald $^{2} \cdot$ Simon Larrosa-Lombardi $^{3}$. \\ Thomas Barchfeld ${ }^{3}$. Ulrich Cleff ${ }^{1}$
}

Received: 12 January 2021 / Accepted: 25 January 2021 / Published online: 4 February 2021

(c) Springer-Verlag GmbH, DE part of Springer Nature 2021

\begin{abstract}
We report on a patient with refractory Myasthenia gravis with acetylcholine receptor antibodies with two prior myasthenic crises suffering from COVID-19 with rapid evolving weakness and respiratory failure. Respiratory failure developed and prolonged mechanical ventilation was necessary. After plasmapheresis, residual, severe generalized and bulbar weakness persisted. Complement inhibition with eculizumab was, therefore, introduced and lead to rapid recovery. In refractory myasthenic crisis individualised therapies could be successful.
\end{abstract}

Keywords COVID-19 · Myasthenia gravis · Complement inhibition · Immunosuppression

\begin{tabular}{|c|c|}
\hline \multicolumn{2}{|c|}{ Abbreviations } \\
\hline AchR & Acetylcholine receptors \\
\hline COVID-19 & Corona virus disease 2019 \\
\hline CT & Computer tomography \\
\hline IvIg & Intravenous immunoglobulins \\
\hline FEES & Functional endoscopic swallowing studies \\
\hline MG & Myasthenia gravis \\
\hline MG-ADL & $\begin{array}{l}\text { Myasthenia gravis Activities of daily } \\
\text { living-Questionnaire }\end{array}$ \\
\hline MGFA & $\begin{array}{l}\text { Myasthenia gravis Foundation of America } \\
\text { scale }\end{array}$ \\
\hline MG-QoL15 & $\begin{array}{l}\text { Myasthenia gravis-Quality of } \\
\text { life-Questionnaire }\end{array}$ \\
\hline MV & Mechanical ventilation \\
\hline PCR & Polymerase chain reaction \\
\hline PEG & Percutaneous enterogastrostomy \\
\hline
\end{tabular}

Ulrich Hofstadt-van Oy

Ulrich.Hofstadt@klinikum-westfalen.de

1 Klinik für Neurologie, Knappschaftskrankenhaus Dortmund, Klinikum Westfalen, 44309 Dortmund, Germany

2 Klinik für Anästhesiologie, Intensivmedizin und Schmerztherapie, Knappschaftskrankenhaus Dortmun, Klinikum Westfalen, 44309 Dortmund, Germany

3 Klinik für Pneumologie, Intensivmedizin und Schlafmedizin, Knappschaftskrankenhaus Dortmund, Klinikum Westfalen, 44309 Dortmund, Germany

4 Fakultät für Gesundheit, Department für Humanmedizin, Universität Witten/Herdecke, 58455 Witten, Germany

\begin{abstract}
PLEX Plasmapheresis
RNS Repetitive nerve stimulation

SARS-CoV2 Severe adult respiratory syndrome Coronavirus 2
\end{abstract}

\section{Introduction}

Myasthenia gravis (MG) is an autoimmune antibody-derived disease of the neuromuscular junction due to impairment of the acetylcholine receptors (AchR) that causes fatiguable ocular, bulbar and limb weakness [1]. Myasthenic crisis is caused by severe weakness of respiratory or bulbar muscles $[2,3]$, and is typically precipitated by poor controlled generalised or bulbar disease, systemic-mostly respiratory-infections, aspiration, surgery or drugs with a known risk for exacerbating myasthenic weakness. Guidelines for management have been established [4].

In the recent global pandemic from novel coronavirus (SARS-CoV2), MG-patients can be considered high risk due to pre-existing weakness of respiratory and bulbar muscles and chronic immunosuppression. In an international registry of 91 patients with MG and Corona virus disease 2019 (COVID-19), $40 \%$ of patients were reported to suffer from a myasthenic crisis and $24 \%$ of patients died [5]. Our case report describes a patient with poorly controlled Ach-R-antibody positive generalised MG with COVID-19 pneumonia, who developed severe bulbar weakness, respiratory failure and sepsis. He was weaned from mechanical ventilation 
(MV) after plasmapheresis (PLEX), but subsequently needed a tracheostomy and nasogastric tube feeding due to severe, generalised weakness and aspiration. After initiation of complement inhibition with eculizumab, removal of tracheostomy and tube feeding were possible and were followed by a good recovery.

\section{Case}

A 62-year-old Caucasian male developed "stiffness of tongue" while eating a late dinner in December 2019, followed by dysphagia over the next days and dysarthria after talking for longer periods, ocular symptoms were absent for the whole course of illness. Six weeks later diurnal fluctuating heaviness of the head with subsequent weakness of the arms developed. 20 weeks after the first symptoms, swallowing was impossible and aspiration pneumonia developed requiring intensive care treatment with MV. After successful weaning, the patient was referred to a neurology department of another hospital where repetitive nerve stimulation (RNS) displayed decrement. AchR-antibodies were highly positive $(90 \mathrm{nmol} / \mathrm{l}$, normal range $<0,2 \mathrm{nmol} / \mathrm{l})$. There was no sign of thymoma on a computer tomography (CT) scan of the chest. The patient developed a second myasthenic crisis with aspiration pneumonia and MV despite therapy with pyridostigmine, steroids up to $80 \mathrm{mg} /$ day and azathioprine. After successful weaning a percutaneous enterogastrostomy tube (PEG) was given to secure oral feeding, azathioprine was administered up to a dose of $150 \mathrm{mg}(2 \mathrm{mg} / \mathrm{kg})$. The patient was still unable to work, neurological deficits were graded according to the Myasthenia gravis Foundation of America scale (MGFA) 4 b [6]. A bleeding duodenal ulcer was treated with high-dose pantoprazole. Previous medical history included residual cardiac insufficiency after myocarditis 15 years earlier, this did not hinder a hiking tour in the Alpes 2 years earlier under a medication with ramipril, telmisartan, carvedilol and torasemide. In August 2020, 6 days before hospital admission, the patient attended a family gathering. Four days later he developed fever, a sore throat, productive cough and increased weakness. On admission he was dyspnoeic and showed severe generalised weakness; rales were auscultated more over the right basal lung. Blood gas analysis was compatible with an acute respiratory failure with hypoxemia and hypercapnia. The patient was transferred to intensive care with strict quarantine measures. Real-time polymerase chain reaction (PCR) for SARS-CoV2 of a nasal/pharyngeal swab was positive. After muscular exhaustion despite non-invasive ventilation, endotracheal intubation and invasive MV was required. Dexamethasone, heparin and piperacilline/tazobactame for aspiration pneumonia were given, azathioprine was stopped. Because of severe respiratory muscle weakness $30 \mathrm{~g}$ intravenous immunoglobulins (ivIg) for five days and high dose continuous pyridostigmine i.v. were given. However, a trial of extubation failed because of respiratory muscle weakness, and the patient underwent endotracheal intubation again. A thoracic CT demonstrated bilateral patchy consolidations and ground-glass opacities compatible with bilateral COVID-19 pneumonia (Fig. 1). Sepsis requiring catecholamines developed. PLEX was considered to be contraindicated and percutaneous dilatational tracheostomy was conducted. Serial sputum SARS-CoV2-PCRs were negative for the first time 15 days after admission. A prolonged septicaemia with Enterobacter cloacae was treated according to a resistogram with high-dose meropeneme, but resolved not before removal of the PEG tube, indicating that the PEG had been the septic focus. Electrodiagnostic studies ruled out signs of polyneuropathy, RNS showed no decrement while high dose pyridostigme was continuously given i.v.. AChRantibodies in serum were $30 \mathrm{mmol} / \mathrm{l}$. PLEX ( 6 cycles 3 times weekly) was begun and prolonged weaning with spontaneous breathing via tracheostomy was successful. Low-dose steroids were added. Severe myalgia required treatment with combination of gabapentin and morphine derivatives. Vaccination against Neisseria meningitidis was carried out. Since immunosuppression with azathioprine and steroids had not stabilised MG before the present myasthenic crisis, B-cell depleting therapy with rituximab $1000 \mathrm{mg}$ was given and repeated after 2 weeks. Functional endoscopic swallowing studies (FEES) showed severe aspiration of saliva and liquids. Severe generalised weakness persisted; the patient had weak truncal control while sitting and was unable to stand (MGFA 5, spontaneous breathing with tracheostomy). After a further 2 weeks without improvement, eculizumab was given $(900 \mathrm{mg}$ weekly for 4 weeks, followed by $1200 \mathrm{mg}$ every 2 weeks) as well as antibiotic coverage with

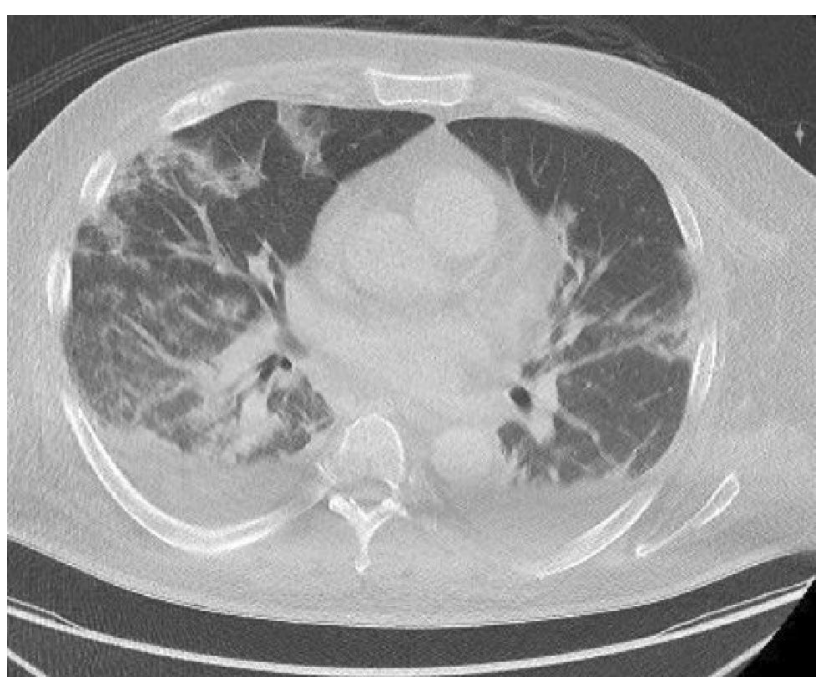

Fig. $1 \mathrm{CT}$ of the chest showing pleural effusions, bilateral patchy consolidations and ground-glass opacities 
ceftriaxone for 2 weeks because of unclear response to Neisseria meningitidis vaccination due to B-cell depletion with rituximab. FEES documented successful clearing of liquids and soft food 1 week after the first dose of eculizumab. Tracheostomy and nasogastric tube could be removed after the second dose of eculizumab. During the following hospital stay and rehabilitation, the patient was able to regain ability to climb stairs and to resume his professional work as a freelancer in own office. Bulbar symptoms such as dysarthria or dysphagia were completely resolved 10 weeks after the start of eculizumab (MGFA 2a). The course of illness with regard to MGFA-classification, Myasthenia gravis-Quality of life-Questionnaire (MG-Qol15) [7], Myasthenia gravis Activities of daily living-Questionnaire (MG-ADL) [8] and therapeutic interventions are shown in Fig. 2.

\section{Discussion}

Refractory MG is characterised by persistent, significant disability despite standard treatment with cholinesterase inhibitors and immunosuppressive strategies such as steroids and azathioprine [9]. However, there is no consensus about the length of time this feature has to be present. In approximately $15 \%$ of patients with MG, a myasthenic crisis with severe weakness of respiratory muscles requires prompt treatment in an intensive care setting. The most common triggers of such crises are systemic or respiratory infections that may occur when respiratory muscle weakness causes atelectasis and dysphagia leads to recurrent aspirations. PLEX and IvIg are preferred treatments of myasthenic crises, along with antibiotics and cholinesterase-inhibitors [4]. The COVID-19 pandemic may involve patients with MG and more than a dozen case reports have been published already. An international registry has collected already 91 cases verifying the grave prognosis for many patients [5], and a consensus statement of a panel of experts has been published [10]. Altering or stopping immunosuppression bears a potential risk for MG patients due to increased disease activity and should therefore be individualised. Our patient had severe, refractory, AchR-antibody positive MG and had survived two myasthenic crisis with MV in the past three months before COVID-19. The initial diagnosis and appropriate therapy were delayed, presumably because he never developed ocular symptoms [11]. Because of increased generalised and bulbar weakness due to COVID-19 IvIg were administered shortly after the initiation of MV with no apparent effect on respiratory muscle weakness. Azathioprine was stopped as the patient developed sepsis; steroids were given knowing their beneficial effect in severe COVID19 infections [12]. After cessation of sepsis, we used PLX, which resulted in spontaneous breathing via tracheostomy, but inability to swallow without aspiration and necessity for nasogastric tube feeding. Since the patient had severe bulbar MG (MGFA 4b) requiring nutrition via PEG before the COVID-19, refractory MG leading to refractory myasthenic crisis was diagnosed and complement inhibition with eculizumab initiated. This led to rapid, persisting recovery with restoration of normal breathing and oral nutrition. It seems unlikely that complement activation by COVID-19 [13] played a role in our patient, because SARS-CoV2-PCR was negative in the 9 weeks before initiation of eculizumab. The role of eculizumab in severe (refractory to PLEX/IvIg) myasthenic crises has to be studied in a randomized controlled trial.

\section{Conclusion}

Treatment of myasthenic crises triggered by COVID-19 needs to be tailored to the individual patient based on the relative severity of MG and COVID-19. Our patient with
Fig. 2 The course of illness in our case with regard to MGFAclassification [6], Myasthenia gravis-Quality of life-Questionnaire (MG-Qol15) [7], Myasthenia gravis Activities of daily living-Questionnaire (MG-ADL) [8] and therapeutic interventions. $M V$ mechanical ventilation, $I v I g$ intravenous immunoglobulins, $P L X$ plasmapheresis

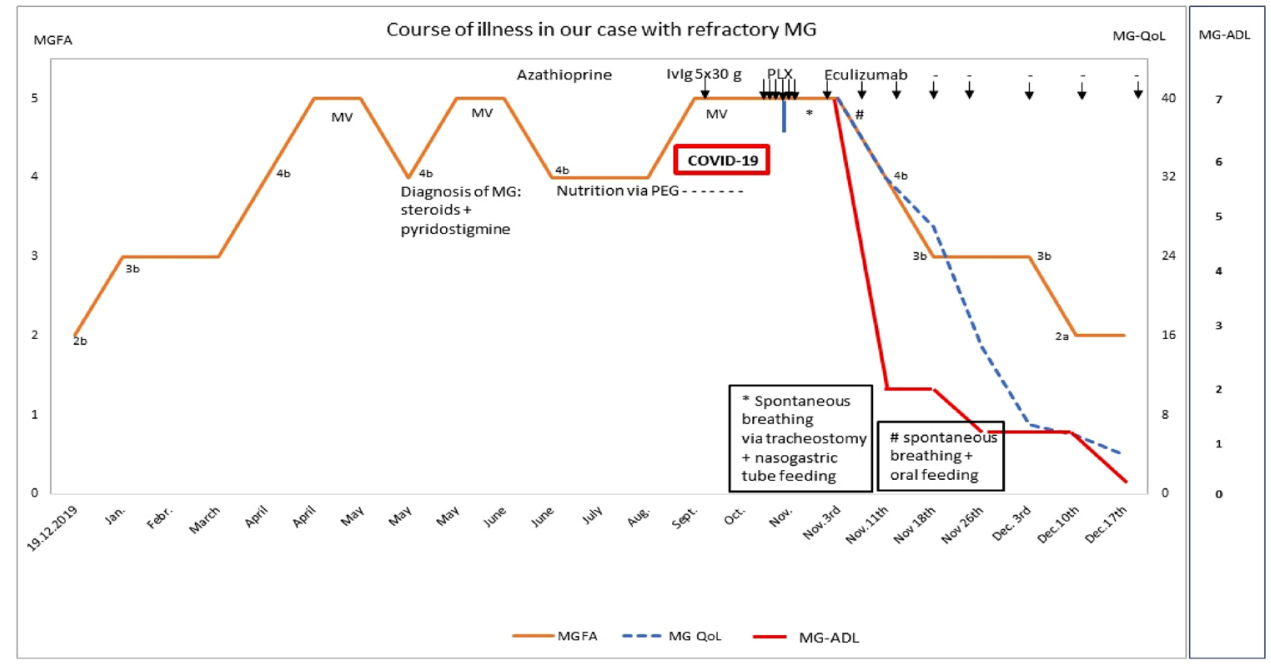


refractory MG, respiratory failure due to COVID-19 pneumonia and persistent septicaemia had a complete recovery (despite MV with prolonged weaning) after receiving an individually tailored sequence of established immunotherapies including IvIg and PLEX and escalation therapy with eculizumab.

Acknowledgements We thank Gail Gerhartz, PhD for linguistic assistance.

Author contributions Clinical care for the patient: UHvO, SS, CK, DO, SLL, TB, UC. Conceptualisation: UHvO. Drafting manuscript, corrections, proofreading: UHvO, SS, CK, DO, SLL, TB, UC. Final edit: UHvO.

\section{Funding None.}

Data availability None.

\section{Compliance with ethical standards}

Conflicts of interest $\mathrm{UHvO}$ reports speaker honoraria from Alexion and Hormosan, the other authors have no conflict of interest to report.

Ethical publication statement We confirm that we have read the Journal's position on issues involved in ethical publication and affirm that this report is consistent with those guidelines.

Informed consent Written informed consent was obtained from the patient.

Consent for publication Available.

\section{References}

1. Gilhus NE (2016) Myasthenia gravis. N Engl J Med $375: 2570-2581$
2. Chaudhuri A, Behan PO (2009) Myasthenic crisis. QJM 2009(102):97-107

3. Neumann B, Angstwurm K, Mergenthaler P et al (2020) Myasthenic crisis demanding mechanical ventilation. A multicenter analysis of 250 cases. Neurology. https://doi.org/10.1212/ WNL.0000000000008688

4. Melzer N, Ruck T, Fuhr P et al (2016) Clinical features, pathogenesis, and treatment of myasthenia gravis: a supplement to the Guidelines of the German Neurological Society. J Neurol 263:1473-1494. https://doi.org/10.1007/s00415-016-8045-z

5. Muppidi S, Guptil JT, Jacob S et al (2020) COVID-19-associated risks and effects in Myasthenia gravis (CARE-MG). Lancet Neurol 19:970-971

6. Jaretzki A, Barohn RJ, Ernstoff RM, Kaminski HJ et al (2000) Myasthenia gravis: recommendations for clinical research standards. Neurology 55:16-23

7. Burns TM, Grouse CK, Wolfe GI et al (2011) The MG-QOL15 for following the health-related quality of life of patients with myasthenia gravis. Muscle Nerve 43:14-18

8. Wolfe GI, Herbelin L, Nations SP, et al. (1999) Myasthenia gravis activities of daily living profile. Neurology. 52: 1487-1487.

9. Mantegazza R, Antozzi C (2018) When myasthenia gravis is deemed refractory: clinical signposts and treatment strategies. Ther Adv Neurol Disord 11:1756285617749134

10. International MG/COVID-19 Working Group, Jacob S, Muppidi S, Guidon A, et al. (2020) Guidance for the management of myasthenia gravis (MG) and Lambert-Eaton myasthenic syndrome (LEMS) during the COVID-19 pandemic. J Neurol Sci. 15: 412:116803. https://doi.org/10.1016/j.jns.2020.116803

11. Yang X, Niu L, Yang C et al (2019) Clinical features of laryngeal myasthenia gravis: a case series. Am J Otolaryng 40:292-296

12. RECOVERY Collaborative Group (2020) Dexamethasone in hospitalized patients with Covid-19-preliminary report. N Engl J Med. https://doi.org/10.1056/NEJMoa2021436

13. Risitano AM, Mastellos DC, Huber-Lang M et al (2020) Complement as a target in COVID-19? Nat Rev Immunol 20:343-344. https://doi.org/10.1038/s41577-020-0320-7 